\title{
Environmental Geochemistry of Reactive Manganese Species: Implications to the Subsurface Redox Dynamics \\ ZIMENG WANG
}

Fudan University

Presenting Author: zimengw@fudan.edu.cn

Manganese (Mn) is the third most abundant transition metal in the Earth's crust and is important to many biogeochemical redox processes. There are emerging evidences of various biogeochemical processes being mediated by Mn redox cycling, which includes uranium, chromium and organic contaminants. While being ignored for a long time, dissolved Mn(III) species have been recently recognized as a significant form of $\mathrm{Mn}$ in redox transition environments. The transient behaviors of dissolved $\mathrm{Mn}$ (III) species make it a representative redox active metastable phase, and its high mobility and turnover may trigger significant subsurface biogeochemical implications. In this presentation, we will share some recent investigations on the environmental geochemistry of reactive manganese species, especially the dissolved $\mathrm{Mn}(\mathrm{III})$ species. Beyond $\mathrm{Mn}$ geochemistry, we will also discuss the general traits of such metastable redox-active species in leveraging geochemical processes with profound impacts. 